\title{
A fast map-making preconditioner for regular scanning patterns
}

\author{
Sigurd K. Næs: ${ }^{\circledast}$ and Thibaut Louis $\dagger^{\dagger}$ \\ Sub-department of Astrophysics, University of Oxford, Keble Road, Oxford, OX1 3RH, UK
}

High-resolution Maximum Likelihood map-making of the Cosmic Microwave Background is usually performed using Conjugate Gradients with a preconditioner that ignores noise correlations. We here present a new preconditioner that approximates the map noise covariance as circulant, and show that this results in a speedup of up to $400 \%$ for a realistic scanning pattern from the Atacama Cosmology Telescope. The improvement is especially large for polarized maps.

\section{INTRODUCTION}

As the resolution and sensitivity of Cosmic Microwave Background (CMB) experiments increase, so do the computational resources needed to analyze their data. Because modern detectors are background-limited, the only way to significantly increase sensitivity is to increase the number of detectors. The last decades have seen an increase from tens of detectors to thousands of detectors in experiments like ACT [8, 14, SPT [3, 5], POLARBEAR [10] and Keck [11, and plans already exist for experiments with $10^{5}-10^{6}$ detectors [1]. Reducing the data from all these detectors into a coherent map of the sky presents a significant computational challenge, and already with 1000-detector-class experiments this step is the most important bottleneck of the data analysis pipeline [8]. It is therefore important to investigate ways to speed up this process.

Three main classes of map-makers are in popular use: Maximum likelihood map-makers [4, 6, 8, [16, 20, 21, which are slow, but produce unbiased, optimally noiseweighted maps; faster but slightly less accurate destripers [9, 19]; and biased and sub-optimal but very fast naive map-makers [16, 17]. The topic of this paper is a method for significantly speeding up maximum likelihood mapmakers.

Assuming a linear detector response, we can model the time-ordered data $d$ via the the linear system

$$
d=P m+n
$$

where $m$ is the pixelized map of the sky, the pointing matrix $P$ is a spars ${ }^{1}$ matrix mapping from pixels to samples, and $n$ is the time-domain noise, which we assume to be gaussian with covariance matrix $N$. The maximum likelihood solution for $m$ is given by the mapmaking equation [20],

$$
\left(P^{T} N^{-1} P\right) m=P^{T} N^{-1} d .
$$

\footnotetext{
* sigurd.naess@astro.ox.ac.uk

$\dagger$ thibaut.louis@astro.ox.ac.uk

1 The pointing matrix will be sparse if we solve for a beamconvolved map. For variable beams or asymmetric beams, one may want to reconvolve to a standard beam as part of mapmaking. This can be done using $P$, at the cost of some of its sparsity. We do not consider this case here.
}

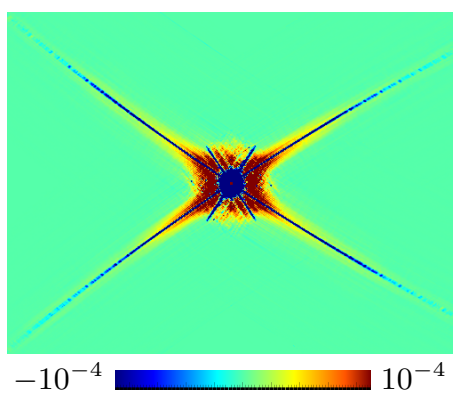

Figure 1 . A $4.2^{\circ}$ by $3.2^{\circ}$ subset of a row from the pixel-space inverse correlation matrix from a patch from ACT (each row in the matrix corresponds to a two-dimensional map). In order to highlight the correlation structure, the color scale is capped at $\pm 10^{-4}$. The significantly correlated area has a quite complicated shape, which is driven by the scanning pattern and focal plane layout.

This is of the form $A x=b$, and while the matrices involved are usually too large to solve by direct inversion, the system is amenable to solution by Preconditioned Conjugate Gradients (PCG) [15] provided a good preconditioner can be found ${ }^{2}$ The most commonly used mapmaking preconditioners are the binned [2, 4, 13] and Jacobi [4, 6, 8] preconditioners. The binned preconditioner $M_{\mathrm{B}}$ approximates the time covariance matrix $N$ as diagonal (i.e. it ignores correlations), which for pointing matrices where only one pixel is hit per sample results in a diagonal pixel-space covariance matrix.

$$
\begin{aligned}
N_{i j}^{-1} & \approx N_{i i}^{-1} \delta_{i j} \text { and } P_{t i} P_{t j} \propto \delta_{i j} \Rightarrow \\
A_{i j} & \equiv P_{t i} N_{t t^{\prime}}^{-1} P_{t^{\prime} j} \approx P_{t i}^{2} N_{t t}^{-1} \delta_{i j} \equiv M_{\mathrm{B}_{i j}}^{-1}
\end{aligned}
$$

The Jacobi preconditioner $M_{\mathrm{J}}$ simplifies one step further, and assumes that every detector has the same variance, $N=a I$, resulting in

$$
A_{i j} \approx a P_{t i}^{2} \delta_{i j} \equiv M_{\mathrm{J}_{i j}}^{-1}
$$

\footnotetext{
2 Without a preconditioner, the number of iterations needed for conjugate gradients is proportional to the condition number of the matrix $A$. By applying a preconditioner $M$, one is effectively solving the system $M A x=M b$. The goal is then to choose $M$ such that $M A$ is as well-conditioned as possible. This can be acchevied if $M \approx A^{-1}$.
} 


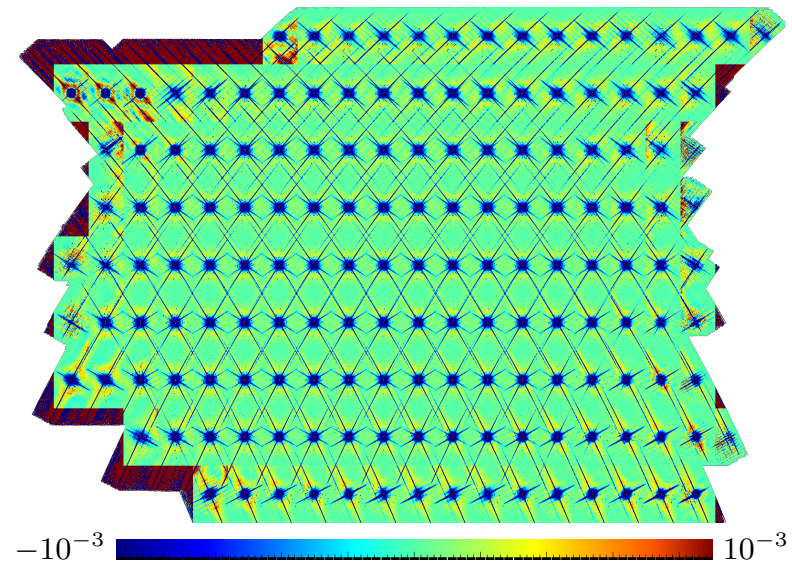

Figure 2. A mosaic of the local correlation structure for a $12^{\circ}$ by $9^{\circ}$ subset of an ACT patch. A set of evenly spaced pixels were chosen as reference points, and for each the correlation in a neighborhood around it was calculated. Each pixel in the map is colored according to its correlation relative to the nearest reference point. The correlation structure is very uniform in the whole central region of the map.

The proportionality factor $a$ is usually set to 1 , as PCG is insensitive to an overall scaling of the preconditioner.

The assumption of independent noise is quite inaccurate. All realistic experiments have at least some timecorrelation in the noise, and usually also correlations between different detectors. Additionally, filters will also generally introduce correlations.

While computing the full, exact $A$ is often too expensive, single rows of it can be computed at the same expense as one $\mathrm{CG}$ iteration:

$$
A_{i j}=A_{i k} \delta_{k j}=\left(A \vec{e}_{i}\right)_{j}
$$

Here $\left(\vec{e}_{i}\right)_{j}=\delta_{i j}$ is the pixel-space basis vector corresponding to pixel $i$. An example of what such a row looks like for the Atacama Cosmology Telescope (ACT) [8] can be seen in figure 1 .

It is clear that the approximation of no correlation is quite inaccurate. However, since the correlation structure is driven by the scanning pattern and relative position of the detectors in the focalplane, the correlation structure should be the same for all pixels which are scanned the same way. ACT, which used long-duration, small-amplitude drift scans, pixels at the same declination but different right ascension will be hit by the same phase of the same scanning motion, and should therefore have the same correlation structure 3

Indeed, that is what measurements show (see figure 2). It may therefore be a good approximation to assume that

3 Circulant correlation is not a good approximation for every experiment. It is suitable for constant elevation dift scans, as employed by ACT, SPT and POLARBear, but we expect it to work poorly for full-sky scanning patterns. every point in the map has the same relative correlation structure, i.e. that the correlation between two points on the sky only depends on their relative position. If this is the case, then it is possible to choose a pixelization where the correlation only depends on the difference between pixel numbers, and hence that the pixel correlation matrix is circulan 4 .

A circulant matrix has the nice property of being diagonal in the frequency domain, which means that it can be computed, stored and applied cheaply, at the cost of a few FFTs. Hence, the constant correlation approximation is promising as a preconditioner for solving the map-making equation.

\section{IMPLEMENTATION}

The inverse pixel covariance matrix $A$ can be decomposed into variance and correlation such that $A=\Sigma^{T} U \Sigma$. Here $\Sigma$ is diagonal (block-diagonal in the case of polarization) in pixel space, and corresponds to a map of the inverse standard deviation per pixel. As per the binned preconditioner, this can be approximated as

$$
\left(\Sigma^{T} \Sigma\right)_{i j}=P_{t i}^{2} N_{t t}^{-1} \delta_{i j}
$$

The correlation matrix $U$ is in general a dense matrix, but as noted above, it can often be approximated as circulant5. Therefore, the constant correlation preconditioner replaces $U$ with a circulant matrix $Q$, such that

$$
A \approx \Sigma^{T} Q \Sigma \equiv M_{\mathrm{C}}{ }^{-1}
$$

This relation can be inverted to give us an expression for $Q$ in terms of $A$,

$$
\begin{gathered}
Q=\Sigma^{T^{-1}} A \Sigma^{-1} \Rightarrow \\
Q_{i j}=\Sigma^{T_{i i}^{-1}}\left(A \vec{e}_{i}\right)_{j} \Sigma_{j j}^{-1} .
\end{gathered}
$$

Since $Q$ is circulant, i.e. $Q_{i j}=Q_{0, j-i}=q_{j-i}$, we have

$$
\left(F Q F^{-1}\right)_{f f^{\prime}}=(F q)_{-f} \delta_{f f^{\prime}}=(F q)_{f}^{*} \delta_{f f^{\prime}}
$$

using forward and backwards Fourier transforms $F_{f j} \equiv$ $\mathrm{e}^{-\frac{2 \pi i j f}{N}}$ and $F_{j f}^{-1} \equiv \frac{1}{N} \mathrm{e}^{\frac{2 \pi i j f}{N}}$, where $N$ is the number of rows in the matrix.

With this in hand, the preconditioner can be applied as

$$
\begin{aligned}
M_{\mathrm{C}} b & =\Sigma^{-1} Q^{-1} \Sigma^{-1^{T}} b \\
& =\Sigma^{-1} F^{-1}(F q)^{*-1} F \Sigma^{-1} b .
\end{aligned}
$$

4 For example, if $\operatorname{corr}\left(\vec{x}_{1}, \vec{x}_{2}\right)=f\left(\vec{x}_{1}-\vec{x}_{2}\right)$, where $\vec{x}$ are coordinates, then a pixelization scheme $\vec{x}=G(p)$, where $p$ is a pixel index and $\mathrm{G}$ is a linear function will fulfill $\operatorname{corr}\left(p_{1}, p_{2}\right)=$ $f\left(G\left(p_{1}-p_{2}\right)\right)$, resulting in a circulant correlation matrix.

5 The matrix will be circulant provided that the correlation structure is position-independent, that a constant offset in each coordinate corresponds to a constant pixel offset, and provided that indices wrap around at the edges. 
$\Sigma^{-1}$ and $(F q)^{*-1}$ can be precomputed, so the cost of applying the preconditioner is simply that of two FFTs and three diagonal matrix multiplications.

The choice of the reference pixel at which the correlation is measured is somewhat arbitrary. We used the pixel nearest the center of the map, but other locations not too close to the edge of the map should also work.

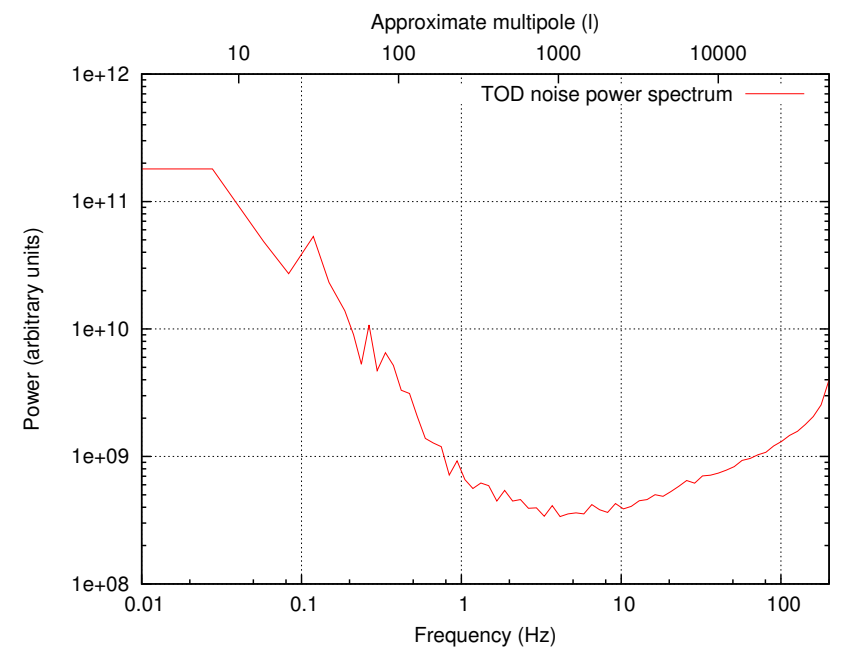

Figure 3. Example TOD noise power spectrum from the simulation, based on noise behavior from ACT. Low frequencies are dominated by atmospheric noise, while the increase at high frequencies is caused by a Butterworth filter.

\section{REGULARIZATION}

While constant correlation is a good approximation for relatively short-scale correlations, it works less well for long-distance correlations, and regions near the edges 6 Applying the preconditioner as described above to realistic cases results in the appearance of large scale modes which change extremely slowly during the subsequent CG iteration.

A way around this is to artificially limit the range of the correlations that are modeled, by multiplying $q$ by a Gaussian. For ACT, a standard deviation of 20 pixels was found to be effective, but this will depend on the scanning pattern, and some experimentation may be needed to find the optimal number.

\section{POLARIZATION}

The previous discussion assumed that each pixel only had a single degree of freedom, e.g. temperature-only

\footnotetext{
${ }^{6}$ Near the edges the telescope must decelerate in order to reverse the scanning direction, which makes the correlation structure different there than in the center.
}

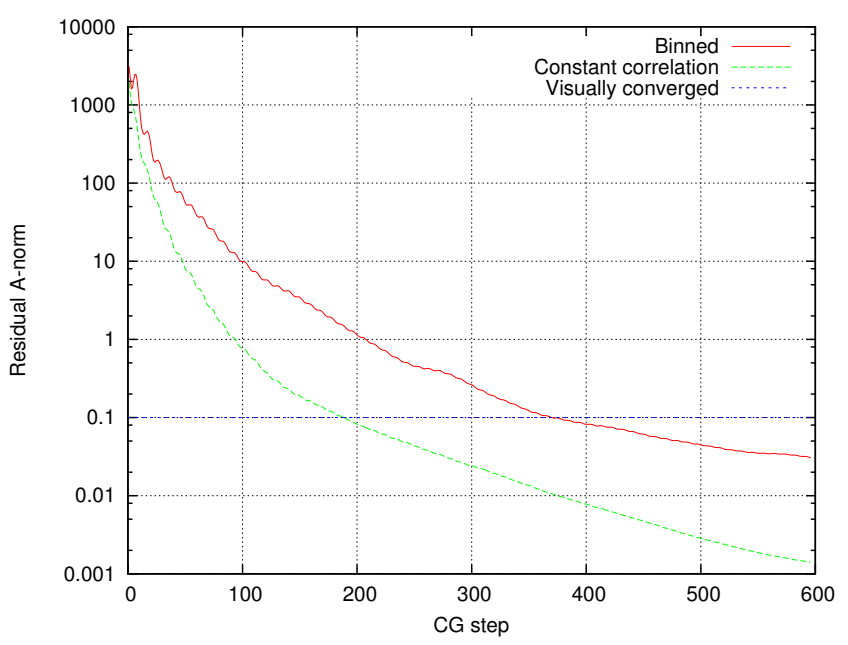

Figure 4. The residual A-norm [18] as a function of iteration number for a simple binned preconditioner and the constant correlation preconditioner. The latter converges roughly twice as fast as the former according to this criterion. The horizontal blue line indicates the level at which the maps have mostly stopped changing visually.

maps of the sky. In the case of polarization, each pixel has several correlated components, typically the Stokes parameters $\mathrm{T}, \mathrm{Q}$ and $\mathrm{U}$ [22, but this can instead be expressed as a larger number of block-correlated singlecomponent pixels. This results in $\Sigma$ being block-diagonal with e.g. one (T,Q,U)-block per physical pixel, while $F q$ becomes a vector of similar blocks. And instead of a single row of $A$ needing to be measured, all the rows corresponding to a given physical pixel now need to be computed (i.e. $\vec{e}_{i \alpha}$ for all components, where Greek indices indicate polarization components). Hence, eq. (10) becomes

$$
Q_{i \alpha j \beta}=\Sigma_{i \alpha i \gamma}^{T-1}\left(A \vec{e}_{i \gamma}\right)_{j \delta} \Sigma_{j \delta j \beta}^{-1}
$$

Aside from that, everything works the same.

\section{TEST SETUP}

We tested the preconditioners on a simulated timeordered data (TOD) based on the scanning pattern for 64 detectors from each of 41715 -minute scans of a subset of ACT's southern patch centered at $\alpha=56^{\circ}, \delta=-53^{\circ}$. Each scan was a constant elevation drift scan with amplitude of $3.5^{\circ}$ in azimuth, and the scan centers were spread over 10 steps in elevation, covering a patch of about $11^{\circ}$ by $8^{\circ}$ degrees. Odd steps in elevation scanned while rising and the even ones when setting. This resulted in most pixels being hit from two directions, and hence the $\mathrm{x}$-shaped correlation pattern seen in figures 1 12 . An example of a noise power spectrum used in the simulation can be seen in figure 3 .

The simulated detectors were polarization-sensitive, with each detector measuring a linear combination $T+$ 

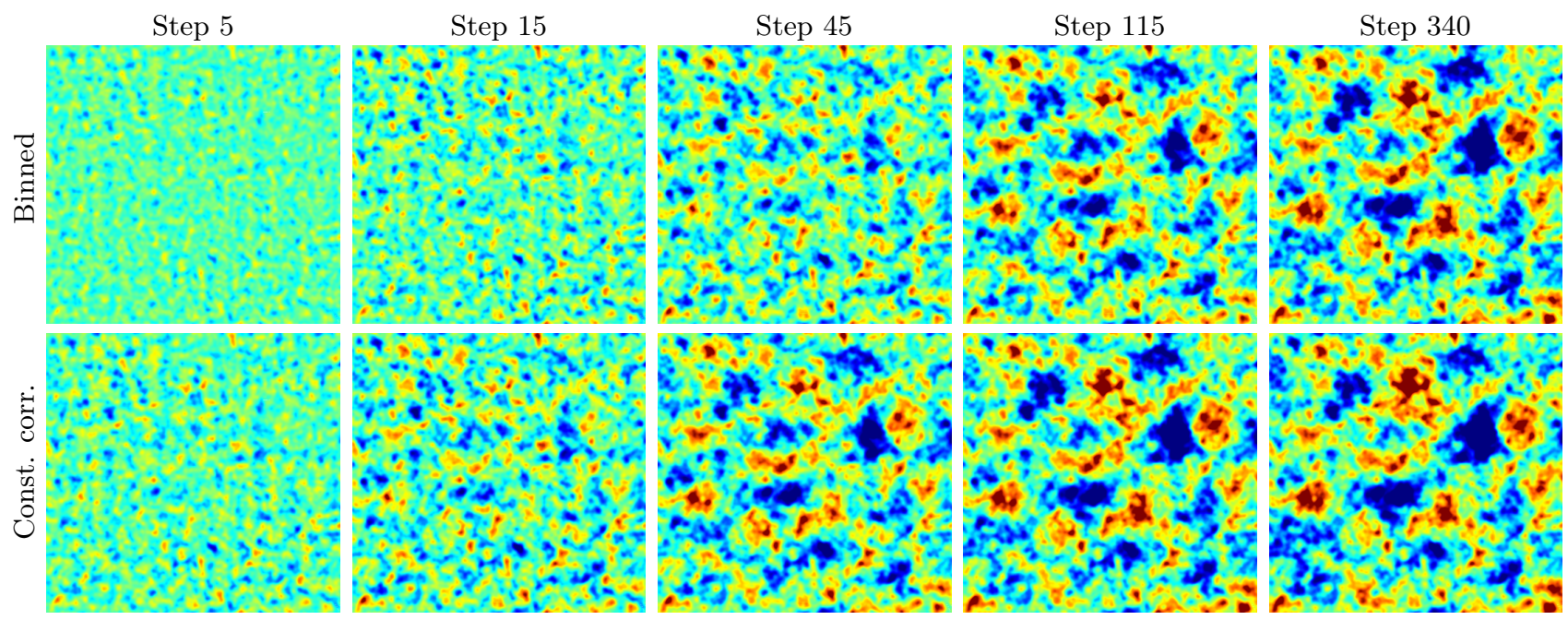

Figure 5. Example temperature maps from the CG solution process for the binned (top) and constant correlation (bottom) preconditioners. The rows correspond to steps 5, 15, 45, 115 and 340 from left to right. The steps are chosen such that the binned map in column $n$ is as similar as possible as the constant correlation map in column $n-1$. We see that the constant correlation preconditioner visually converges about 3 times faster than the binned one. The maps have been cropped for compactness of presentation.

Step 15

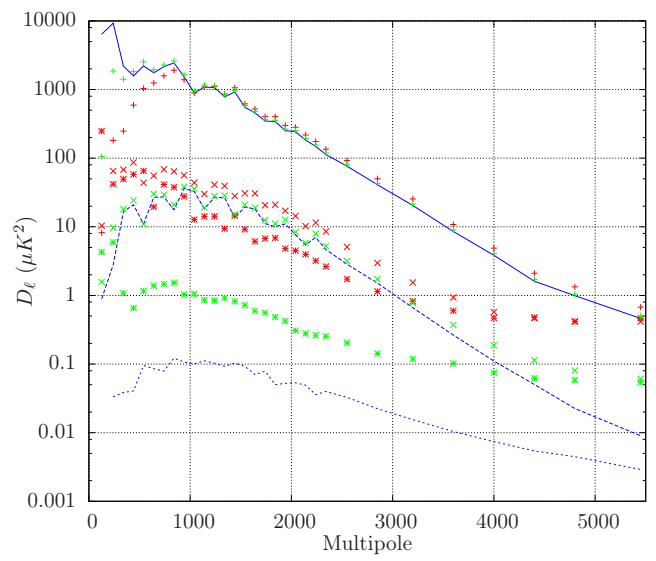

Step 70

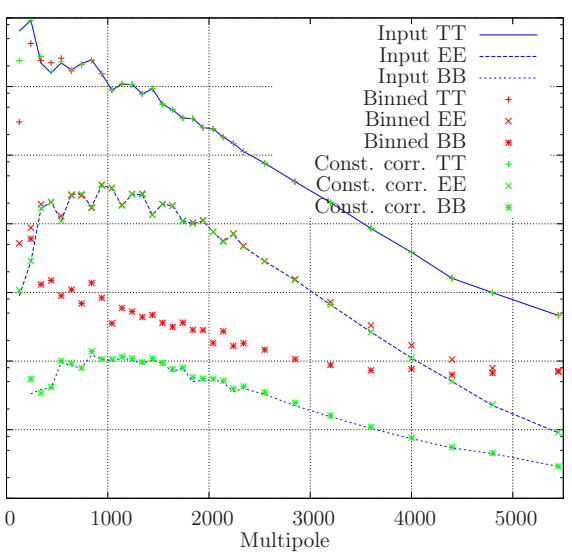

Step 250

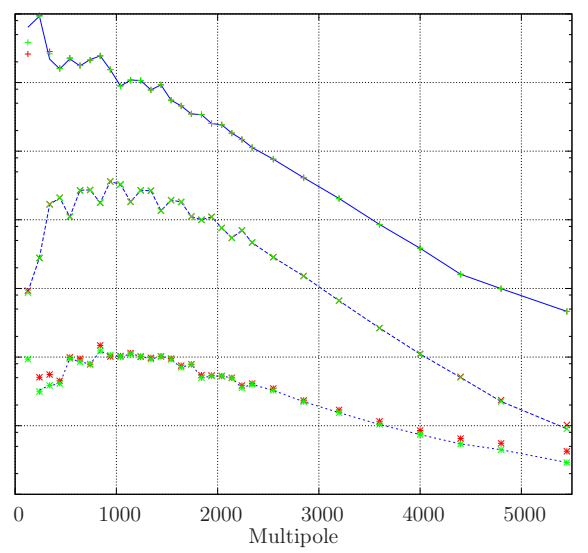

Figure 6. Comparison of the input (blue), binned (red) and constant correlation (green) power spectra at CG steps 15 (left), 70 (middle) and 250 (right). Unlike the map-space plots in figure 5 where only the large-scale convergence is visible, we can here clearly see the convergence at all scales. Small-scale convergence is much slower for EE and BB than for TT, and form the bottleneck for the CG solver if we ignore the $\ell<500$ modes. The constant correlation preconditioner is $3-5$ times faster than the binned one here. This is quantified more precisely in figure 9

$\cos (2 \psi) Q+\sin (2 \psi) U$ of the local radiation field, with each detector having a different, randomly chosen detector angle $\psi$. While an ACT-like noise model, including the effects of atmosphere and inter-detector noise correlations was assumed in the map-making step, no noise was added to the simulated TOD in order to allow the convergence to be studied all the way to the highest multipole: 7. For the same reason, the simulated input CMB did not include a beam, and was pixelated at the same

7 This is valid since the convergence rate of PCG is mostly inde- resolution as the output map, in order to avoid subpixel noise.

We then solved the map-making equation for this data set using PCG, first using the binned preconditioner described in equation (4), and then the constant correlation approximation described in this paper. Each was run for

pendent of the noise level of the right-hand side after the first few iterations 13. However, with higher noise, higher CG errors also become acceptable, so the number of iterations needed for CG errors to be subdominant will be smaller for realistic noise levels. 
600 CG iterations, with intermediate maps being output for every 5 steps.

\section{RESULTS}

The constant correlation preconditioner visually converges roughly 3 times faster than the binned one, as shown for the temperature map in figure 5. Likewise, the residual A-norm 18] from the CG solver (shown in figure 44) also shows a significant improvement in convergence: roughly a factor 2 according to this metrie 8

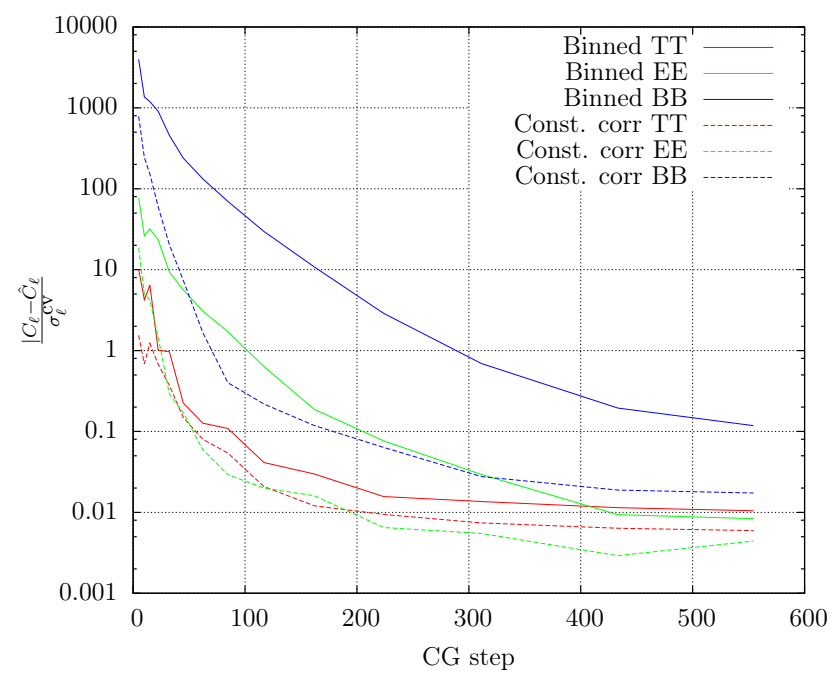

Figure 7. An example of the convergence of a single bin in the power spectrum, in this case that centered on $\ell=3200$. This shows the absolute difference between the recovered spectrum $C_{\ell}$ and the spectrum $\hat{C}_{\ell}$ of the input map in units of cosmic variance $\sigma_{\ell}^{\text {cv }}$, as a function of the CG step in cosmic variance units. Shown are curves for TT (red), EE (green) and $\mathrm{BB}$ (blue) for the binned (solid) and constant correlation (dashed) preconditioners. The trend lines are based on binned averages of the errors for many individual CG steps. This binning reduces the jitter, and makes the trend lines suitable for measuring the time needed to converge to a given level.

However, neither of these tests take into account the fact that not all scales in the map are equally interesting. To remedy this, figure 6 compares the binned and constant correlation power spectra with that of the simulated input map. These spectra were computed using

\footnotetext{
8 The A-norm $\|x\|_{A}$ of a vector $x$ is defined as $\sqrt{x^{T} A x}$, where $A$ is $P^{T} N^{-1} P$ in our case. The error A-norm after $i$ CG steps is $\left\|x_{i}-x\right\|_{A}$, where $x_{i}$ is our estimate after $i$ steps, and $x$ is the true map. When the true $x$ is unknown, $\left\|x_{i}-x\right\|_{A}$ can be estimated as $\left\|x_{i}-x\right\|_{A} \approx \sum_{j=i}^{i+d-1} \gamma_{j}\left\|r_{j}\right\|^{2}$, where $r_{j}$ and $\gamma_{j}$ are two internal variables in the CG algorithm at step $j$, and $d$ is is an integer that controls the accuracy of the estimate (4 in our case).
}

the method described in [12. On large scales $(\ell<500)$, this tells the same story as the maps did: The larger the scale, the more slowly it converges, with the constant correlation preconditioner being about 3 times faster than the binned one. Somewhat surprisingly, a similar phenomenon occurs at the small scales. For $\ell>2000$, higher $\ell$ results in slower convergence, and this is especially prominent for the $\mathrm{EE}$ and $\mathrm{BB}$ power spectra. On all scales, however, the constant correlation preconditioner appears to converge several times faster than the binned one.

In order to quantify the convergence more precisely, we consider the time at which the absolute error in a given multipole-bin reaches 0.1 times cosmic variance in that bin. While somewhat arbitrary, this choice ensures that CG errors are guaranteed to be sub-dominant in the power spectrum, regardless of the noise properties of the actual experiment. Figure 7 shows the convergence of $\mathrm{TT}$, EE and BB for both preconditioners for a typical multipole-bin. The overall trend for each component is an initial rapid fall followed by a slower decay, with both being significantly faster for the new preconditioner, particularly for the polarization spectra.

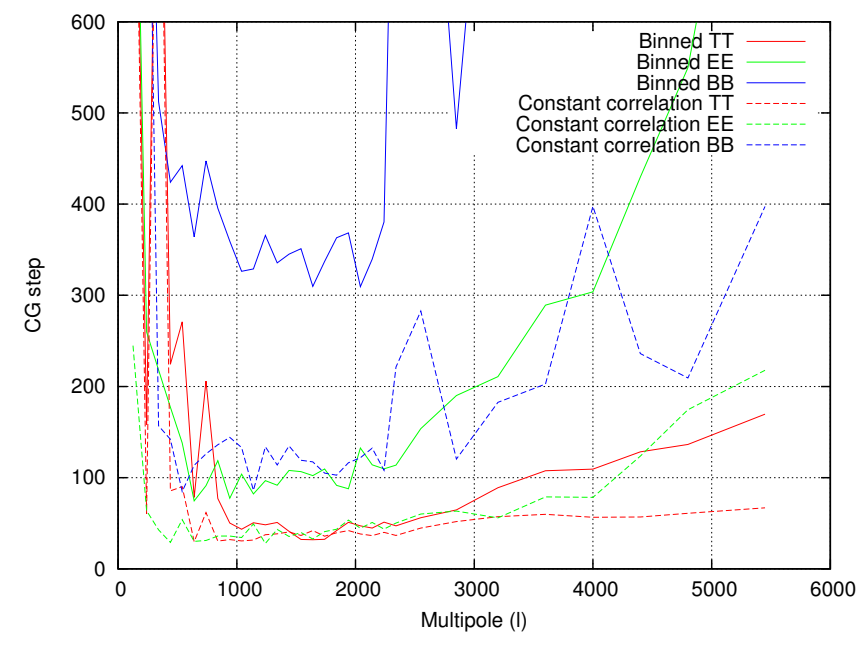

Figure 8. The number of CG steps needed for each multipolebin to converge to 0.1 times cosmic variance, for each of TT (red), EE (green) and BB (blue) for the binned (solid) and constant correlation (dashed) preconditioners.

We found that binning the errors in bins of $\sim 50$ conjugate gradients steps and using linear interpolation between these bins resulted in a robust estimate of when each spectrum bin reaches the convergence criterion. The resulting convergence times can be seen in figure 8 , and confirm our earlier finding that the largest and smallest scales converge more slowly. The figure also highlights how much trouble the binned preconditioner has with the EE and especially BB spectra, where it performs much more poorly relative the constant correlation preconditioner than we see for the TT spectrum. We speculate that this is due to the $\mathrm{X}$-shaped correlation 
structure introduced by our scanning pattern. In binned maps, which ignore the correlations, this introduces spurious $\mathrm{X}$-shaped patterns in both $\mathrm{Q}$ and $\mathrm{U}$, corresponding to spurious signal in both $\mathrm{E}$ and $\mathrm{B}$. With the constant correlation preconditioner, these are partially corrected because some of the correlation structure is taken into account.

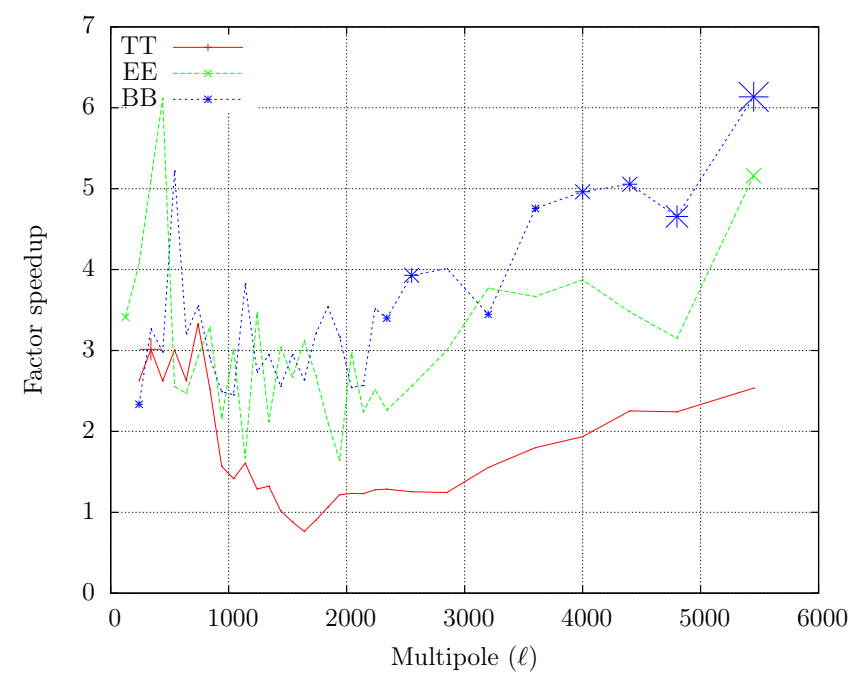

Figure 9. The ratio of the convergence times to 0.1 times cosmic variance for the binned and constant correlation preconditioners, per multipole bin for each of TT, EE and BB. For TT, the speedup is typically between $20 \%$ and $200 \%$, while for BB the speedup ranges from $200 \%$ at large scales to about $400 \%$ at small scales, with $\mathrm{EE}$ being intermediate. Large points here indicate cases where the binned preconditioner did not converge to 0.1 times cosmic variance in time. For these points, the comparison was performed at the lowest threshold where both converged.

We summarize the performance characteristics of the new preconditioner in figure 9, which shows its relative speed gain vs. the baseline binned preconditioner. Depending on $\ell$, we have a speedup ranging from $20 \%$ to $200 \%$ for TT, from $150 \%$ to $300 \%$ for EE, and from $200 \%$ to $400 \%$ for $\mathrm{BB}$, with the greatest relative improvement happening at the scales that converge most slowly.

\section{SUMMARY}

The structure of a CMB map's pixel covariance matrix is determined by the noise properties of the time-ordered data and the scanning pattern of the telescope. For constant elevation drift scans like those employed by ACT, SPT and POLARBEAR, this results in an approximately circulant covariance. We have developed a new preconditioner for conjugate gradient solutions of the map-making equation which exploit this property by deconvolving the correlations in harmonic space, an operation which is very cheap due to the Fourier representation of a circulant matrices being diagonal.

For a realistic scanning pattern and noise model the preconditioner results a speedup of $20 \%$ to $200 \%$ for temperature and $150 \%$ to $400 \%$ for polarization compared to a binned preconditioner.

Convergence speed might potentially be further improved by allowing the correlation pattern to change slowly across the map, for example by decomposing the map into overlapping tiles, and applying the constant correlation preconditioner separately to each tile, followed by a merging operation. Our preliminary attempts at such a tiled preconditioner have however not been able to beat the simple constant correlation approximation presented here.

\section{ACKNOWLEDGMENTS}

The authors would like to thank Jo Dunkley and Johannes Noller for useful discussion and suggestions, and Jon Sievers for testing the preconditioner in another mapmaker. We also thank the ACT collaboration for access to internal ACT data used in the simulations. Computations were performed on the gpc supercomputer at the SciNet HPC Consortium. SciNet is funded by: the Canada Foundation for Innovation under the auspices of Compute Canada; the Government of Ontario; Ontario Research Fund - Research Excellence; and the University of Toronto. SN and TL are supported by ERC grant 259505 .
[1] Abazajian, K. N., Arnold, K., Austermann, J., et al. 2013, ArXiv e-prints, arXiv:1309.5383

[2] Ashdown, M. A. J., Baccigalupi, C., Balbi, A., et al. 2007, A\&A, 467, 761

[3] Austermann, J. E., Aird, K. A., Beall, J. A., et al. 2012, in Society of Photo-Optical Instrumentation Engineers (SPIE) Conference Series, Vol. 8452, Society of PhotoOptical Instrumentation Engineers (SPIE) Conference Series

[4] Cantalupo, C. M., Borrill, J. D., Jaffe, A. H., Kisner, T. S., \& Stompor, R. 2010, ApJS, 187, 212

[5] Carlstrom, J. E., Ade, P. A. R., Aird, K. A., et al. 2011,
PASP, 123, 568

[6] de Gasperis, G., Balbi, A., Cabella, P., Natoli, P., \& Vittorio, N. 2005, A\&A, 436, 1159

[7] Doré, O., Teyssier, R., Bouchet, F. R., Vibert, D., \& Prunet, S. 2001, A\&A, 374, 358

[8] Dünner, R., Hasselfield, M., Marriage, T. A., et al. 2013, ApJ, 762, 10

[9] Keihänen, E., Kurki-Suonio, H., \& Poutanen, T. 2005, MNRAS, 360, 390

[10] Kermish, Z. D., Ade, P., Anthony, A., et al. 2012, in Society of Photo-Optical Instrumentation Engineers (SPIE) Conference Series, Vol. 8452, Society of Photo-Optical 
Instrumentation Engineers (SPIE) Conference Series

[11] Kernasovskiy, S., Ade, P. A. R., Aikin, R. W., et al. 2012, in Society of Photo-Optical Instrumentation Engineers (SPIE) Conference Series, Vol. 8452, Society of Photo-Optical Instrumentation Engineers (SPIE) Conference Series

[12] Louis, T., Næss, S., Das, S., Dunkley, J., \& Sherwin, B. 2013, ArXiv e-prints, arXiv:1306.6692

[13] Natoli, P., de Gasperis, G., Gheller, C., \& Vittorio, N. 2001, A\&A, 372, 346

[14] Niemack, M. D., Ade, P. A. R., Aguirre, J., et al. 2010, in Society of Photo-Optical Instrumentation Engineers (SPIE) Conference Series, Vol. 7741, Society of PhotoOptical Instrumentation Engineers (SPIE) Conference Series
[15] Press, W. H., Teukolsky, S. A., Vetterling, W. T., \& Flannery, B. P. 2007, Numerical Recipes 3rd Edition: The Art of Scientific Computing, 3rd edn. (Cambridge University Press)

[16] QUIET Collaboration, Araujo, D., Bischoff, C., et al. 2012, ApJ, 760, 145

[17] Schaffer, K. K., Crawford, T. M., Aird, K. A., et al. 2011, Astrophys. J., 743, 90

[18] Strakos, Z., \& Tichy, P. 2008, ETNA, 13, 56

[19] Sutton, D., Zuntz, J. A., Ferreira, P. G., et al. 2010, MNRAS, 407, 1387

[20] Tegmark, M. 1997, ApJL, 480, L87

[21] Traficante, A., Calzoletti, L., Veneziani, M., et al. 2011, MNRAS, 416, 2932

[22] Zaldarriaga, M., \& Seljak, U. 1997, Phys. Rev. D, 55, 1830 